\title{
BMJ Open Pain sensitisation and the risk of poor outcome following physiotherapy for patients with moderate to severe knee osteoarthritis: protocol for a prospective cohort study
}

\author{
Helen O'Leary, ${ }^{1}$ Keith M Smart, ${ }^{2}$ Niamh A Moloney, ${ }^{3}$ Catherine Blake, ${ }^{1}$ \\ Catherine M Doody ${ }^{1}$
}

To cite: O'Leary $\mathrm{H}$, Smart KM, Moloney NA, et al. Pain sensitisation and the risk of poor outcome following physiotherapy for patients with moderate to severe knee osteoarthritis: protocol for a prospective cohort study. BMJ Open 2015:5:e007430.

doi:10.1136/bmjopen-2014007430

- Prepublication history for this paper is available online. To view these files please visit the journal online (http://dx.doi.org/10.1136/ bmjopen-2014-007430).

Received 11 December 2014 Revised 21 May 2015 Accepted 22 May 2015

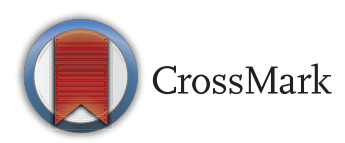

For numbered affiliations see end of article.

Correspondence to

Helen O'Leary;

helen.oleary@ucdconnect.ie

\section{ABSTRACT}

Introduction: Pain is the dominant symptom of knee osteoarthritis $(\mathrm{OA})$, and recent evidence suggests factors outside of local joint pathology, such as pain sensitisation, can contribute significantly to the pain experience. It is unknown how pain sensitisation influences outcomes from commonly employed interventions such as physiotherapy. The aims of this study are, first, to provide a comprehensive description of the somatosensory characteristics of people with pain associated with knee OA. Second, we will investigate if indicators of pain sensitisation in patients with knee osteoarthritis are predictive of non-response to physiotherapy.

Methods and analysis: This is a multicentre prospective cohort study with 140 participants. Eligible patients with moderate to severe symptomatic knee osteoarthritis will be identified at outpatient orthopaedic and rheumatology clinics. A baseline assessment will provide a comprehensive description of the somatosensory characteristics of each participant by means of clinical examination, quantitative sensory testing, and validated questionnaires measuring pain and functional capacity. Participants will then undergo physiotherapy treatment. The primary outcome will be non-response to physiotherapy on completion of the physiotherapy treatment programme as defined by the Osteoarthritis Research Society International treatment responder criteria. A principal component analysis will identify measures related to pain sensitisation to include in the predictive model. Regression analyses will explore the relationship between responder status and pain sensitisation while accounting for confounders.

Ethics and dissemination: This study has been approved by St James' Hospital/AMNCH Research Ethics Committee and by the St Vincent's Healthcare Group Ethics and Medical Research Committee. The results will be presented at international conferences and published in a peer review journal.

Trial registration number: NCT02310945.
Strengths and limitations of this study

To the best of our knowledge, this is the first study prospectively examining the effects of pain sensitisation on physiotherapy outcomes.

- Strengths of this proposed study include the relatively large sample for the comprehensive assessment procedure involved; the use of a broad range of validated measures to study pain processing; and the gathering of our own reference QST data from healthy volunteers.

- This study will focus on tests to identify features of pain sensitivity on the basis of their utility and practicality in the clinical setting. However, these criteria necessitate the exclusion of certain tests, such as thermal pain thresholds, that are difficult to carry out reliably in the clinical setting in a limited time frame.

- As this is a clinically based observational study, there is likely to be variation in the duration and type of physiotherapy interventions. The findings of this research may identify clinical and psychophysiological variables predictive of a poor response to physiotherapy that might usefully inform subsequent studies aimed at targeting such variables in an attempt to optimise patients' outcomes.

\section{INTRODUCTION}

Knee osteoarthritis (OA) is a prevalent joint disease that causes a huge burden of pain, disability and loss of productivity worldwide. ${ }^{1-3}$ With ageing populations and increasing obesity, the prevalence of $\mathrm{OA}$ is rising, thus, its timely and effective management is a priority within healthcare. ${ }^{34}$

The pathophysiology of knee OA pain is complex. Altered processing of nociceptive inputs at peripheral, spinal and higher brain centres may help explain discrepancies between pain severity, and the degree of structural and pathological abnormalities 
in OA. $^{5-7}$ Central sensitisation is described as an increased responsiveness of nociceptive neurons in the central nervous system to their normal or subthreshold afferent input. ${ }^{8}$ It can have widespread effects such as general pain hypersensitivity, while other regional manifestations of central sensitisation include spread of pain sensitivity to normal tissue, an exaggerated response to a noxious stimulus and pain after the end of a stimulus. ${ }^{9}$ Furthermore, peripheral proinflammatory mediators and neuropeptides in knee OA can sensitise nociceptors in the affected knee, lowering their threshold for activation. ${ }^{10}$ This increased responsiveness of nociceptive neurons is referred to as peripheral sensitisation. ${ }^{8}$

Both peripheral and central sensitisation, clinically referred to as pain sensitisation, can contribute to painful knee OA. Pain sensitisation may be useful as a clinical construct to alert clinicians to patients with a potentially upregulated nociceptive state, and is proposed to contribute to an enhanced, persistent and more widespread pain response. ${ }^{11}$ Recent studies in knee OA have found the presence of increased pain sensitivity at remote sites, enhanced temporal summation (TS) and hypersensitivity to various stimuli, to be associated with reports of more severe symptoms. ${ }^{5} 612$ However, these cross-sectional studies linking features of sensitisation to greater levels of pain and disability do not explore if pain sensitivity has any prognostic implications.

There is also some evidence to suggest that increased pain sensitivity negatively affects treatment outcomes. In painful musculoskeletal conditions such as shoulder impingement and lateral epicondylalgia, widespread pressure pain sensitivity and thermal hyperalgesia, have been linked with a poorer prognosis. ${ }^{13}{ }^{14}$ Surgical outcomes for knee osteoarthritis may also be affected, with the presence of increased pain sensitivity prior to total knee replacement associated with more persistent pain after surgery. ${ }^{15} 16$

Although joint replacement is considered an effective treatment for end-stage knee OA, the majority of patients are managed conservatively. Physiotherapy is the widely recommended conservative treatment approach for knee OA. ${ }^{17}$ Existing studies of prognosis in knee OA have focused on demographic and psychological variables. ${ }^{18-20}$ While it has been suggested that central pain processing may contribute significantly to the clinical pain experience in some people with knee $\mathrm{OA},{ }^{21}$ no longitudinal studies have explored the potentially negative prognostic impact of pain sensitisation on outcomes in response to physiotherapy. In whiplash-associated disorders, the presence of sensory hypersensitivity and cold hyperalgesia has been shown to reduce the likelihood of a positive response to physiotherapy treatment. ${ }^{22}$ Thus, it is conceivable, but currently unproven, that patients with knee OA with evidence of pain sensitisation have poorer outcomes following physiotherapy.

One obstacle to investigating the implications of pain sensitisation is reliably identifying it in the clinical setting. Owing to the complexity of pain mechanisms, it is inadvisable to rely on any single test to reflect peripheral and central pain mechanisms. ${ }^{23}{ }^{24} \mathrm{~A}$ multitissue assessment using a multimodal stimuli approach has been advocated, ${ }^{25}$ and will be adopted in this study. The association between key features of pain sensitisation and clinical characteristics in knee OA have been previously investigated. Our study will be the first to prospectively explore the effect of key features of pain sensitisation on physiotherapy outcomes in knee OA.

This study will investigate the extent to which pain sensitisation predicts non-response to physiotherapy in patients with knee OA. Identifying clinical and psychophysical features of pain sensitisation in knee OA predictive of a poor response to physiotherapy might help inform the management of such patients. It may encourage clinicians to consider additional or alternative interventions aimed at reducing such pain sensitisation, and optimise outcomes.

\section{Study aims}

The main aims of the study are, first, to provide a comprehensive description of the somatosensory characteristics of people with pain associated with knee OA (by means of quantitative sensory testing (QST) and validated questionnaires measuring pain, functional capacity and quality of life) and second, to investigate if the presence of pain sensitisation at baseline is predictive of non-response to physiotherapy treatment as defined by treatment responder criteria.

We hypothesise that the presence of pain sensitisation will predict a non-response to physiotherapy treatment compared to patients without evidence of pain sensitisation.

\section{METHODS AND ANALYSIS}

\section{Study design}

A multicentre observational cohort study with assessments at baseline, post-treatment and at 6 months will be conducted. Following the baseline assessment for features of pain sensitisation, all participants will receive usual physiotherapy care. The relationship between pain sensitisation and outcomes in terms of pain and disability will be explored through regression analysis.

\section{Setting}

The study will be undertaken in the physiotherapy outpatient departments of three large publicly funded university teaching hospitals in Dublin, Ireland.

\section{Participants}

Patients with symptomatic knee OA referred for physiotherapy treatment by a hospital consultant or clinical specialist physiotherapist will be eligible for inclusion. Full details of inclusion/exclusion criteria are summarised in table 1.

At the time of recruitment, knee pain must be the participant's primary musculoskeletal problem for which they are seeking treatment, and physiotherapy must be 
Table 1 Study inclusion and exclusion criteria

\begin{tabular}{|c|c|}
\hline \multicolumn{2}{|l|}{ Inclusion criteria } \\
\hline Diagnosis & $\begin{array}{l}\text { Knee osteoarthritis based on American College of Rheumatology clinical criteria }{ }^{26} \text { and confirmed } \\
\text { by radiographic findings }\end{array}$ \\
\hline Age & Over 50 years \\
\hline Pain duration & Knee pain for at least 6 months \\
\hline Severity & Average pain over past week rated as moderate or severe by the patient \\
\hline Medication & $\begin{array}{l}\text { Willing to abstain from simple analgaesics, NSAIDs, weak opioids or medications that combine } \\
\text { these, for } 24 \mathrm{~h} \text { prior to testing }\end{array}$ \\
\hline Consent & Willing and able to give full consent \\
\hline \multicolumn{2}{|l|}{ Exclusion criteria } \\
\hline Pathology & $\begin{array}{l}\text { Lumbar or cervical radiculopathy, systematic inflammatory disease, positive screen for diabetic } \\
\text { neuropathy, neuropathic pain }\end{array}$ \\
\hline Past medical history & $\begin{array}{l}\text { Previous surgery or disease of the peripheral or central nervous system, sensory loss secondary to } \\
\text { chemotherapy or radiotherapy, fibromyalgia, chronic fatigue syndrome }\end{array}$ \\
\hline Cognitive ability & $\begin{array}{l}\text { Cognitive or psychiatric disorder interfering with ability to fully consent or cooperate with } \\
\text { assessment }\end{array}$ \\
\hline Other treatment & Injection or physiotherapy treatment for knee joint within previous 3 months \\
\hline Medication & Taking antidepressant or anticonvulsant medication, strong opioids \\
\hline
\end{tabular}

the main treatment being undertaken over the study period. Participants recruited at physiotherapy-led musculoskeletal assessment clinics will be screened for eligibility by the clinical specialist physiotherapist. The principal investigator will screen patients on the physiotherapy waiting list over the telephone.

\section{Healthy controls}

Healthy participants are defined as people with no current pain or chronic pain problems in the past year. Forty age-matched and gender-matched controls will be recruited. Controls aged 50-65 years will be recruited mainly from the staff and student population of University College Dublin, while pain-free controls aged 65-80+ years will be recruited from the general population. Recruitment will be purposive in order to fill the quotas in terms of age and gender. The controls will provide reference data for QST results.

\section{Investigator}

The principal investigator (HOL), collecting all baseline and follow-up data, will be a senior physiotherapist with 12 years clinical experience. The same investigator will carry out all tests and is trained in using QST.

\section{Recruitment procedure}

A consecutive sample of patients with knee OA with moderate/severe knee pain will be recruited. Between October 2014 and September 2015, potentially eligible participants will be identified at musculoskeletal assessment clinics and from physiotherapy outpatient waiting lists. A feasible recruitment rate is estimated at 12 patients per month with recruitment continuing until the specified numbers are achieved. Potential participants will be screened over the telephone and asked to choose a categorical pain descriptor (mild/ moderate/severe). Patients who rate their symptoms as mild (average symptoms over the past week) will be excluded. Those who meet the other inclusion criteria will be asked to attend for an assessment prior to starting physiotherapy treatment. Written informed consent will be obtained before enrolment in the study. Figure 1 represents the flow of participants through the study.

\section{Physiotherapy management}

Physiotherapy treatment will be in line with current clinical guidelines for the management of knee OA. ${ }^{27}$ Treatment will typically involve between four and six physiotherapy appointments. In some cases, treatment may take the format of a small group exercise intervention. A workshop led by the principal investigator will be held at each recruitment site prior to the start of the study where physiotherapists will receive an update on clinical guidelines and current best evidence on management of knee OA. This will standardise treatment to some degree, but intervention will be individualised at the discretion of the treating physiotherapist and in consultation with the patient.

\section{Assessment}

Baseline assessment

A schematic view of the outcome measures recorded at baseline and follow-up is presented in table 2. Each assessment will take approximately $1 \mathrm{~h}$ to complete. Some questionnaires will be posted and completed in advance by participants.

\section{Follow-up assessment}

The primary end point will be at completion of physiotherapy treatment, this time point is estimated to be, on average, at 3 months. Physiotherapy administration staff will alert the principal investigator when a 


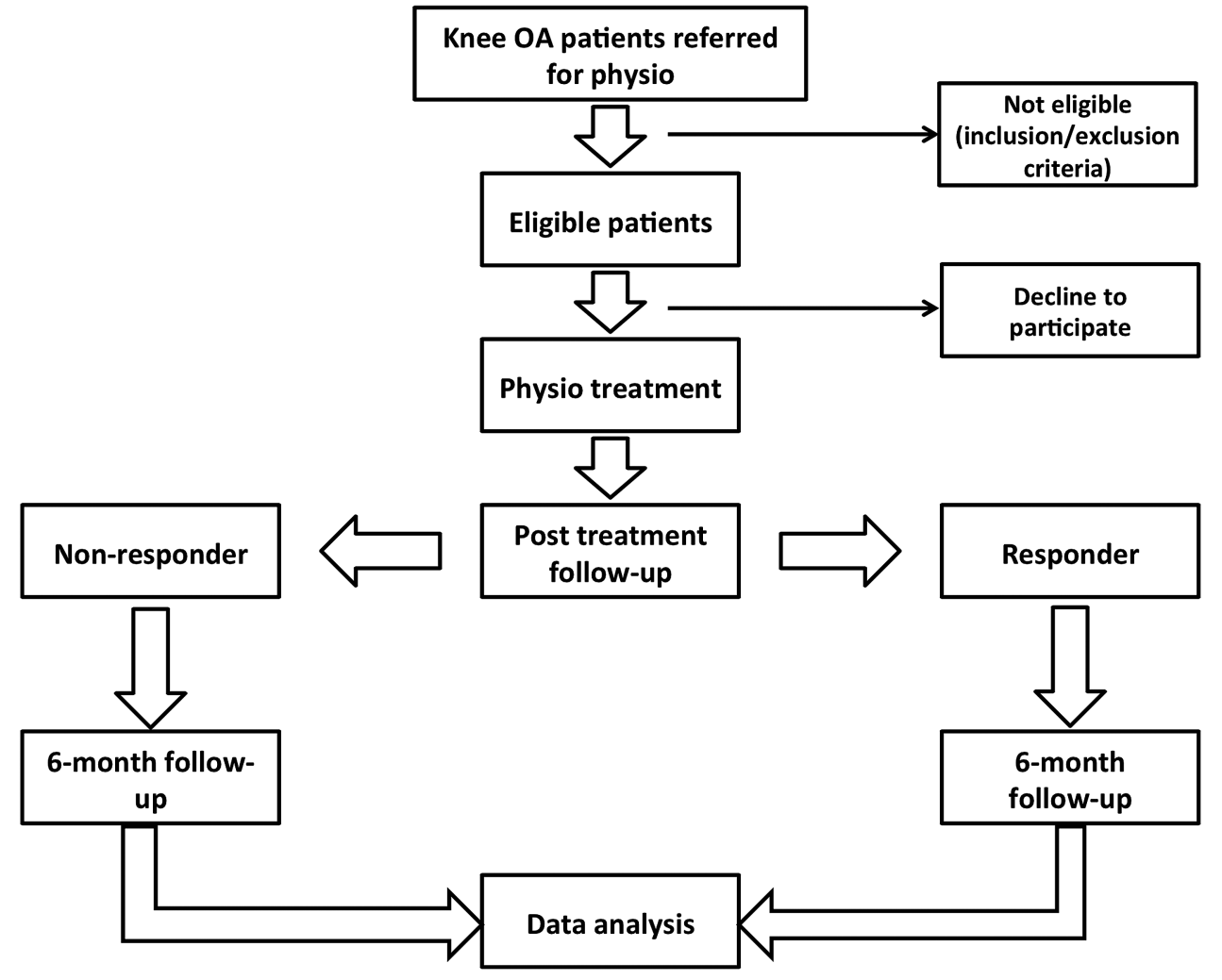

Figure 1 Flow of participants through the study.

participant is discharged. Thereupon pain, disability and global rating of change will be assessed by means of a postal questionnaire. Information will also be recorded on use of co-interventions or any change in medication for knee pain. This follow-up questionnaire will be administered 1 week following discharge from physiotherapy.

Six months after enrolment into the study, participants will complete a postal questionnaire assessing pain and function. They will exit the study at this point.

\section{Assessment procedures and minimising bias}

Studies support the reliability of QST measures where protocols are standardised, and both the tester and participant are carefully instructed. ${ }^{25}$ Standardised assessment procedures will be followed in this study. At each location, testing will be undertaken in the same temperature controlled room by the same investigator, using a single set of testing devices. Each session will begin by familiarising participants with standardised test procedures. $^{28}{ }^{29}$ Physical testing will be performed by the investigator prior to in-depth subjective assessment or without knowledge of questionnaire scores. Test order is important, ${ }^{30}$ and a predetermined sequence will be used beginning with non-noxious stimuli. Conditioned pain modulation (CPM) can induce a residual effect and will be the final test. ${ }^{31}$ With bilateral symptoms, the more painful knee will be selected; if both are equally symptomatic, the right knee will be tested. Where shoulder pain is present unilaterally the opposite forearm will be used for testing.

\section{Primary outcome variable}

The main outcome is response to physiotherapy treatment, and this will be determined using a set of responder criteria by the Outcome Measures in RheumatologyOsteoarthritis Research Society International (OMERACTOARSI) ${ }^{32}$ Non-response to physiotherapy will be the designated categorical dependent variable on which the subsequent regression analyses will be based.

The responder criteria will be applied to the relevant data gathered at post-treatment follow-up, and are summarised in figure 2. For application of these criteria, pain and function will be measured with the subscales of the Western Ontario and McMasters University Score Osteoarthritis Index (WOMAC LK 3.0). Validity and reliability of these subscales are well established, including for postal surveys. ${ }^{33-35}$ Global rating of change will be measured with a seven-point Likert scale. This scale captures relevant change by asking the patient about any improvement or deterioration that has occurred with physiotherapy treatment. ${ }^{36}$ Two points on the scale represents a $20 \%$ improvement.

\section{Secondary outcome variables}

Pain assessment

The following valid and reliable measures of pain will be recorded: Pain Intensity Numerical Rating Scale (Pain 
Table 2 Outcome measures and collection points

\begin{tabular}{|c|c|c|c|}
\hline Domain & Variable & Instrument for data collection & Collection points \\
\hline Demographics & $\begin{array}{l}\text { Age, gender, educational attainment, } \\
\text { employment status, marital status }\end{array}$ & Baseline assessment questionnaire & Baseline \\
\hline \multirow[t]{6}{*}{ Pain } & Self-reported pain & WOMAC pain subscale & $\begin{array}{l}\text { Baseline, } \\
\text { post-treatment*, } \\
6 \text { months }\end{array}$ \\
\hline & Pain intensity & Numerical rating scale & Baseline, 6 months \\
\hline & Location and quality & Knee Pain Map & Baseline \\
\hline & Neuropathic pain symptoms & Modified PainDETECT & Baseline \\
\hline & Characteristics of osteoarthritic pain & $\begin{array}{l}\text { Intermittent and Constant } \\
\text { Osteoarthritis Pain Instrument }\end{array}$ & Baseline \\
\hline & Widespread pain & $\begin{array}{l}\text { Body chart } \\
\text { Manual tender point count }\end{array}$ & Baseline \\
\hline Function & Self reported function & WOMAC function subscale & $\begin{array}{l}\text { Baseline, } \\
\text { post-treatment, } \\
6 \text { months }\end{array}$ \\
\hline Quality of life & Health-related quality of life & EQ-5D 5L & Baseline \\
\hline $\begin{array}{l}\text { Central Sensitisation } \\
\text { Symptoms }\end{array}$ & $\begin{array}{l}\text { Non-musculoskeletal central } \\
\text { sensitisation symptoms }\end{array}$ & Central Sensitization Inventory & Baseline \\
\hline \multirow{8}{*}{$\begin{array}{l}\text { Quantitative sensory } \\
\text { testing }\end{array}$} & Light touch & Von Frey filaments & Baseline \\
\hline & Vibration & Graded tuning fork & Baseline \\
\hline & Pain pressure thresholds & Pressure algometry & Baseline \\
\hline & Dynamic allodynia & Brush stroke & Baseline \\
\hline & static allodynia & Von Frey Filaments & \\
\hline & Thermal hyperalgesia & Thermo-rollers & Baseline \\
\hline & Temporal summation & $\begin{array}{l}\text { Repetitive mechanical stimuli with } \\
\text { weighted pinprick }\end{array}$ & Baseline \\
\hline & Conditioned pain modulation & $\begin{array}{l}\text { Cold pressor test (conditioning } \\
\text { stimulus) and PPTs (test stimulus) }\end{array}$ & Baseline \\
\hline \multirow[t]{3}{*}{ Confounding Variables } & Obesity & $\begin{array}{l}\text { Ratio of waist circumference to } \\
\text { height }\end{array}$ & Baseline \\
\hline & Depressive symptoms & $\begin{array}{l}\text { Centre for Epidemiologic Studies } \\
\text { Depression Scale }\end{array}$ & Baseline \\
\hline & Comorbidities & $\begin{array}{l}\text { Self Administered Comorbidity } \\
\text { Questionnaire }\end{array}$ & Baseline \\
\hline \multirow[t]{5}{*}{$\begin{array}{l}\text { Management-related } \\
\text { variables }\end{array}$} & Treatment adherence & $\begin{array}{l}\text { Sports Injury Rehabilitation } \\
\text { Adherence Scale }\end{array}$ & During treatment \\
\hline & & Patient attendance ratio & During treatment \\
\hline & & $\begin{array}{l}\text { Home exercise compliance } \\
\text { assessment }\end{array}$ & During treatment \\
\hline & $\begin{array}{l}\text { Treatment type and duration, } \\
\text { adverse effects }\end{array}$ & Therapist record sheet & During treatment \\
\hline & Medication use and co-interventions & Follow-up questionnaire & $\begin{array}{l}\text { Post-treatment, } \\
6 \text { months }\end{array}$ \\
\hline Treatment outcome & Response to treatment & $\begin{array}{l}\text { OARSI responder criteria; WOMAC } \\
\text { pain and function subscales, global } \\
\text { rating of change }\end{array}$ & Post-treatment \\
\hline
\end{tabular}

*Post-treatment assessment will be at approximately 3 months.

NRS) will measure participant's average pain intensity over the previous 7 days. ${ }^{37} 38$ The Knee Pain Map will be used to record more detailed information about the location and quality of knee pain. ${ }^{39}$ Widespread pain is defined for this study according to the American College of Rheumatology classification criteria using pain drawings marked by participants on a body manikin. ${ }^{40}$ Widespread pain is associated with more severe knee pain and functional decline. ${ }^{41} 42$
Pain and quality-of-life questionnaires

Modified painDETECT-questionnaire (MPD-Q)

This questionnaire will record any neuropathic component to participants' symptoms. It has been previously used for the screening of neuropathic pain-like symptoms in knee OA in an elderly cohort. $^{43}$ Participants with more neuropathic pain-like symptoms (scores $>12 / 38$ ) are more likely to have signs of central sensitisation. ${ }^{44}$ 


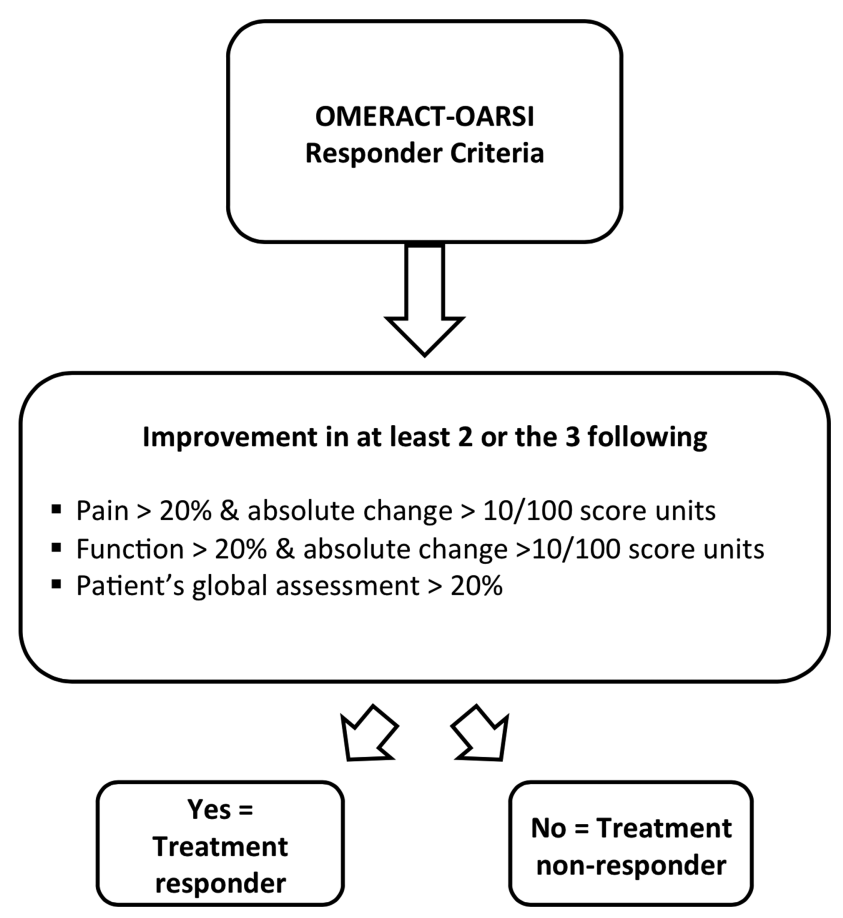

Figure 2 Set of responder criteria. ${ }^{32}$

\section{Intermittent and constant osteoarthritis pain instrument (ICOAP)}

This validated questionnaire assesses various facets of both intermittent and constant pain for the knee, including effects on sleep and quality of life, degree of frustration and worry associated with the pain. ${ }^{45}$ Two predictability items will be administered to capture unpredictable spontaneous pain, thought to have the greatest impact on participant well-being. ${ }^{46}$

\section{Central Sensitization Inventory (CSI)}

This self-report inventory has preliminary validity and reliability and assesses for symptoms not related to the musculoskeletal system but common to central sensitisation syndromes. ${ }^{478}$ Good sensitivity $(81 \%)$ and specificity $(79 \%)$ values were found with a cut-off score of 40 (of 100) to identify patients with symptoms of central sensitisation. $^{48}$

\section{EuroQoL 5Q-5D-5L}

The EQ-5D is a frequently used generic quality-of-life instrument, designed by the EuroQoL group. ${ }^{49}$ A modified form has been developed with an enlarged number of possible answers to avoid a ceiling effect. ${ }^{50}$ The EQ-5D has acceptable reliability and validity when used in patients with knee $\mathrm{OA}^{51}$

\section{Quantitative sensory testing (QST)}

QST is a psychophysiological measure of perception in response to external stimuli of controlled intensity. ${ }^{29}$ This QST protocol will make reference to the wellestablished German Neuropathic Pain Consortium (DFNS) protocol, ${ }^{29}$ and will utilise clinical QST methods recommended by the International Association for the Study of Pain. ${ }^{28}$ This current study's assessment protocol aims to be more accessible using tools that are relatively inexpensive and adaptable to the clinical setting. ${ }^{52}$ Test sites used will be as follows-Site 1: on the medial or lateral knee joint line, depending on where the patient indicates their greatest pain $(3 \mathrm{~cm}$ medial to medial edge of patella or corresponding site laterally); Site 2: over ipslateral tibialis anterior muscle $(5 \mathrm{~cm}$ distal to the tibial tuberosity); Site 3: on the contralateral forearm ( $5 \mathrm{~cm}$ distal to the lateral epicondyle of the humerus on the volar aspect). Somatosensory abnormalities over the area of Site 2 tibialis anterior are thought to provide evidence of spreading sensitisation from the symptomatic knee. Changes at Site 3 could indicate more widespread sensitisation at a generalised level in the central nervous system, ${ }^{11}$ although this cannot be concluded definitively as other explanations for abnormal QST results outside the knee are also possible, for example, patients with knee OA frequently have multisite pain. ${ }^{41}$

\section{Light touch}

Mechanical detection thresholds will be tested using a set of von Frey filaments (Opti-hair2-Set, Marstock, Germany). Monofilaments beginning with the smallest diameter will be applied to the skin. Light touch threshold $\left(\mathrm{g} / \mathrm{mm}^{2}\right)$ will be recorded as the last filament $\left(\mathrm{g} / \mathrm{mm}^{2}\right)$ that can be perceived. Test-retest reliability of this method for knee OA has been established. ${ }^{53}$

\section{Mechanical allodynia}

Dynamic mechanical allodynia will be assessed by lightly stroking the knee and forearm with a brush stroke three times (Senselab Brush No 5, Somedic). Static allodynia will be assessed using von Frey filaments. The presence of mechanical allodynia will be recorded if this nonnoxious stimulation evokes a sensation of pain. ${ }^{54}$

\section{Thermal hyperalgesia}

Thermal rollers with predetermined temperatures of $25^{\circ} \mathrm{C}$ (cold) and $40^{\circ} \mathrm{C}$ (warm) (Senselab Rolltemp Somedic) will be used to detect thermal hyperalgesia at the forearm and knee. ${ }^{52}$ Participants will be asked to report if the thermal sensation is perceived as painful when the rollers are passed lightly over the skin.

\section{Vibration}

Vibration detection threshold will be measured with a graded tuning fork (Rydel-Seiffer, $64 \mathrm{~Hz}$ ) placed over three bony prominences (ulnar styloid, patella and medial malleolus). Vibration detection threshold is determined as a mean disappearance threshold of the vibrations on an $8 / 8$ scale with the stimulus repeated three times. ${ }^{29}$

\section{Pressure pain thresholds (PPTS)}

Pressure will be applied using an electronic digital algometer with a probe size of $1 \mathrm{~cm}^{2}$ (SomedicAB, 
Sweden), and an application rate of $30 \mathrm{kPa} / \mathrm{s}$. The participant will be instructed to press an automatic cut-off button when the first sensation of pressure pain is perceived. A cut-off point is set at $1000 \mathrm{kPa}$. PPTs will be measured three times at the three test sites; the first measurement will be excluded and the mean of the last two measurements will be used for analysis. Test-retest reliability using this technique has been demonstrated in patients with knee OA. ${ }^{55}$

\section{Mechanical temporal summation}

The participant will assign a pain rating to a single stimulus by a weighted pinprick (MRC Systems $256 \mathrm{mN}$ ) and estimate an overall pain rating for a series of 10 pinprick stimuli of the same intensity (1/s applied within an area of $\left.1 \mathrm{~cm}^{2}\right) .{ }^{29}$ This procedure will be applied twice on a marked area at the forearm and knee site. To get a value for TS, the mean pain rating of the two pinprick trains will be calculated minus the mean pain rating of the two single stimuli. ${ }^{56}$

\section{Conditioned pain modulation}

The cold presser test is recommended for assessment of CPM in the clinical setting. ${ }^{57}{ }^{58}$ The test stimulus will be PPTs, while the conditioning stimulus will be cold immersion. PPTs will be recorded as outlined above. With the participant seated comfortably, the opposite arm to that used for PPT testing will be immersed in a bath of icy water $\left(4^{\circ} \mathrm{C}\right.$ monitored by thermometer) up to the elbow for $60 \mathrm{~s}$. Participants unable to tolerate the water bath will rate their pain before withdrawing the arm (aim for at least 5 on NRS). ${ }^{31}{ }^{57}$ Retesting of PPTs on the contralateral forearm will take place immediately after immersion. The PPT values after the cold pressor test will be divided by PPTs recorded before the test. A value of $\leq 1$ will be taken to reflect no CPM effect.

\section{Manual tender point examination}

Tender points are typically identified according to the American College of Rheumatology criteria for fibromyalgia. ${ }^{59}$ Points can be reliably identified by application of pressure with the thumb pad of the tester's dominant hand to 18 designated sites for $4 \mathrm{~s}$ until $4 \mathrm{~kg}$ (450 kPa) of pressure is achieved. Those points where pressure causes pain are summed to give a tender point total. Detecting tender points with digital palpation has good intra-rater reliability, and is considered a useful clinical measure for deep tissue hyperalgesia. ${ }^{60}$

\section{Patient adherence}

Patient adherence to treatment is thought to be an important determinant of clinical outcome in knee OA. ${ }^{17}$ The physiotherapist will calculate an attendance ratio for each patient. ${ }^{62}{ }^{63}$ Additionally, the Sports Injury Rehabilitation Adherence Scale (SIRAS) will be used to measure physiotherapists' perceptions of their patient's rehabilitation adherence at each clinic appointment. In addition to its proven psychometric properties, the SIRAS has been shown to be a reliable scale for use in clinical physiotherapy. ${ }^{64}$ The Home Exercise Compliance Assessment is a widely used self-report method of assessment to measure adherence. At each physiotherapy appointment, participants will record the extent to which they adhered to home exercises and physical activity advice since their previous clinic appointment. ${ }^{63}$

\section{Confounding variables}

Potentially confounding sociodemographic parameters, including age, gender, marital status, employment status and educational level, will be recorded on a standardised form. ${ }^{20} 6566$ Obesity will be measured by recording participants' waist circumference to height ratio. ${ }^{67}$

Other factors known to predict poor outcome in knee OA or influence pain and disability will be accounted for, including multiple comorbidities and depressive symptoms. ${ }^{18}$ The Centre for Epidemiologic Studies Depression Scale is a valid and reliable measure of depression in community-dwelling elderly, and a score $>16$ is considered indicative of depressive symptoms. ${ }^{68}$ For each of these variables, the method of assessment is detailed in table 2 .

The presence of widespread pain is another potential confounder; ${ }^{41}$ however, it could also be a mediator between pain sensitisation and QST measures such as lowered PPTs, remotely. For this reason, the regression model will not be adjusted for this variable.

Radiographic severity of knee OA is poorly correlated with self-reported pain and pain sensitisation. ${ }^{5}{ }^{6}$ For these reasons, X-ray results will not be included in the analysis. ${ }^{69}$

\section{Data analysis plan}

In order to describe the somatosensory characteristics, z-scores will be calculated for individual patients with $\mathrm{OA}$. The control group will provide reference data for QST results and enable calculation of a standardised $\mathrm{z}$-score using the following formula: $\mathrm{z}=$ (value $_{\text {participant- }}$ - mean $\left._{\text {controls }}\right) / \mathrm{SD}_{\text {controls }}{ }^{29}$ Calculating the z-score for each QST modality and body site facilitates the comparison of QST results with healthy control subjects independent of the unit of measure. For data analysis where cut-off points are required, z-scores outside the 10th and 90th centile (or 1.28 SDs of the mean) will be classified as abnormal.

To address the first aim, descriptive statistics will be calculated for all outcome measures at baseline, including for all continuous variables, means, SDs, or medians with ranges of scores; and for categorical variables, frequencies and percentages. In summarising descriptive QST data, z-score calculations and cut-off points will determine the prevalence of somatosensory abnormalities in pressure pain thresholds, mechanical detection threshold and vibration threshold. Somatosensory abnormalities such as allodynia, or cold hyperalgesia, will be classified as either present or absent.

Initial analyses for the second aim will be exploratory to compare symptom profiles between people who 
respond to treatment and treatment non-responders. These will be categorised by the OMERACT-OARSI responder criteria as described previously. Categorical variables will be analysed using $\chi^{2}$ tests. Multivariate analysis of variance will be used to compare continuous normally distributed variables between responders and non-responders, and, from this, variables associated with responder status will be identified. The Mann-Whitney test will be used for comparison of variables that are not normally distributed. In cases where data are missing, multiple imputations will be applied. A sensitivity analysis will be carried out to assess if this changes the results.

A principal component analysis (PCA) will be used to determine which variables relating to sensitisation (light touch, vibration, allodynia, cold hyperalgesia, PPT arm, PPT knee, PPT tibia, TS arm, TS knee, CPM) to include in the predictive model. ${ }^{70}$ PCA may facilitate data reduction, as some variables related to pain sensitisation may be highly correlated while some sensory modalities may represent distinct individual dimensions of pain perception. ${ }^{71}$ Prior to conducting PCA, the suitability of the data for this type of analysis will need to be assessed. Components with an eigenvalue $>1.0$ from PCA will be subsequently entered into the regression model investigating predictive factors for non-response to physiotherapy.

A logistic regression model will be developed to predict non-response to physiotherapy treatment with 'treatment non-responder' (yes/no) as the dependent variable. Variables will be chosen for inclusion in the first model iteration if they are found to be associated with nonresponder status in univariate analysis with a threshold of $\mathrm{p}<0.05$, or if their inclusion is supported by previous literature. The model will be adjusted for predetermined variables such as age, gender, socioeconomic status, depressive symptoms, treatment adherence and comorbidities. ${ }^{1806566}$ Results of the PCA will be entered into the regression model. It is anticipated that the regression model will accommodate a maximum of seven variables with statistical significance accepted if $\mathrm{p}<0.05$. The best fitting and most parsimonious model will be selected as the final iteration, and cross-validation of the predictive model will be performed. ${ }^{72}$ A secondary subanalysis will explore the ability of some individual QST modalities to predict nonresponse to physiotherapy if entered into the regression model as single variables.

Data will be analysed using SPSS V.20 (SPSS, Chicago, Illinois, USA) and R V.3.0.2.

\section{Sample size}

The sample size is calculated based on the number of anticipated explanatory variables planned for inclusion in the logistic regression model. It is expected that a maximum of seven explanatory variables will be included and 10 cases, that is, non-responders, will be allowed for each explanatory variable. ${ }^{73}$ It is estimated that $60 \%$ of patients will be classified as non-responders to physiotherapy by the OMERACT-OARSI criteria. $^{74}$ Recruiting 140 participants while allowing for a $15 \%$ loss to follow-up should ensure adequate numbers.

\section{Dropouts}

All participants will be followed up on discharge from physiotherapy. Patients who discontinued treatment will have the opportunity to provide follow-up data. Patients will be considered lost to follow-up if they do not complete the primary outcome measure post-treatment.

\section{DISSEMINATION}

Fully informed written consent will be obtained, and patients incapable of giving full consent will not be recruited.

The study findings will be presented at national and international conferences, and will be published in peerreviewed journals.

\section{DISCUSSION}

To the best of our knowledge, this is the first study to examine the effects of pain sensitisation on clinical outcomes in response to physiotherapy in patients with knee OA. The research is exploratory in nature, and some limitations are outlined below. For the purposes of this clinical research, the term pain sensitisation will be utilised, however, the lack of a widely accepted definition and criteria for identifying pain sensitisation is acknowledged as a limitation.

It is recognised that some experimental pain modalities, such as enhanced TS and facilitatory CPM, are often interpreted as hallmarks of pain sensitisation, but these can also occur in healthy populations. ${ }^{565}$ It is important to acknowledge this natural variability in responses and the likelihood of these tests capturing false positives. To account for this, no individual QST modality will be used as a stand-alone measure of pain sensitisation, and QST results will be compared with normative data.

The QST protocols originally developed for assessing neuropathic pain are lengthy. ${ }^{29}$ However, if somatosensory testing is to be incorporated into routine clinical practice, it must be both time and cost efficient. ${ }^{52} 75$ This study will focus on tests to identify features of pain sensitivity based on their utility and practicality in the clinical setting. However, these criteria necessitate the exclusion of certain tests, such as thermal pain thresholds, that are difficult to carry out reliably in the clinical setting in a limited time frame.

The central concern of this study relates to alterations in pain processing, and any potential relationship with poorer prognosis in knee OA. A range of clinical, psychological and sociodemographic predictors of poor outcome have been identified. ${ }^{76-78}$ Incorporating all these variables is not feasible, and would make for an unacceptably long participant assessment and complex analytical model. Nonetheless, the most important predictor variables will be accounted for in the analysis. 
This clinically based observational study does not aim to investigate the effects of specific physiotherapy treatments. It will observe people undergoing usual physiotherapy, and variation in the intervention is to be expected. Nonetheless, an attempt to standardise care to some degree will be made by using current evidence-based guidelines, and keeping a record of the physiotherapy intervention and adherence for each participant.

Recruiting the participant sample from the secondary care setting will limit the ability to generalise the study findings to patient populations in primary care. The generalisability is further limited by only including patients with moderate or severe knee symptoms. This limitation was deemed necessary in order to include sufficient numbers of patients likely to have features of sensitisation, as we know pain sensitisation is related to pain intensity. ${ }^{5}$ Furthermore, optimising management in secondary care is a priority; this is where Irish patients usually access not just physiotherapy services but more invasive treatments such as joint injection or surgery.

The analyses reported in this study will be exploratory and generate rather than confirm hypotheses about pain sensitisation and physiotherapy for knee OA. It is acknowledged that widespread pain hypersensitivity and somatosensory abnormalities can arise from a host of complex and interacting neurophysiological, psychological and immunological processes. ${ }^{21}$

Given its relatively short follow-up period, we cannot infer causality directly from our data with regard to pain sensitisation and physiotherapy outcomes. Nonetheless, it may point to a relationship worthy of further investigation in order to better understand pain mechanisms in knee OA, and optimise physiotherapy outcomes in the future.

\section{Author affiliations \\ ${ }^{1}$ School of Public Health, Physiotherapy and Population Science, University College Dublin, Dublin, Ireland \\ ${ }^{2}$ Physiotherapy Department, St Vincent's University Hospital, Dublin, Ireland ${ }^{3}$ Discipline of Physiotherapy, Department of Health Sciences, Macquarie University, Sydney, New South Wales, Australia}

Acknowledgements The authors would like to acknowledge the Physiotherapy Departments of St James's Hospital, St Vincent's University Hospital and Tallaght Hospital, Dublin, for agreeing to facilitate this research.

Contributors HO, CMD, KMS and NAM assisted with the protocol design. CMD, KMS, NAM and $\mathrm{HO}$ procured the project funding. CB provided statistical advice. All authors provided feedback on drafts of the paper and approved the final manuscript.

Funding This work was supported by a Research Fellowship for Healthcare Professionals from the Health Research Board, Ireland. Grant number: HPF/ 2013/449

\section{Competing interests None declared.}

Ethics approval St James' Hospital/AMNCH Research Ethics Committee (ref no 2013/11/Chair) and by the St Vincent's Healthcare Group Ethics and Medical Research Committee (ref no HOL/9414), and will be conducted in accordance with the Helsinki Declaration.

Provenance and peer review Not commissioned; externally peer reviewed.
Data sharing statement It is the intention of the study group that once the study is completed and the research articles arising from the study have been published, any data that is fully anonymised and not sensitive would be made open access. However, because this intention was not declared in the original ethical applications, this action would be subject to approval by the relevant research ethics committees.

Open Access This is an Open Access article distributed in accordance with the Creative Commons Attribution Non Commercial (CC BY-NC 4.0) license, which permits others to distribute, remix, adapt, build upon this work noncommercially, and license their derivative works on different terms, provided the original work is properly cited and the use is non-commercial. See: http:// creativecommons.org/licenses/by-nc/4.0/

\section{REFERENCES}

1. Kingsbury SR, Gross HJ, Isherwood G, et al. Osteoarthritis in Europe: impact on health status, work productivity and use of pharmacotherapies in five European countries. Rheumatology (Oxford) 2014;53:937-47.

2. Losina E, Walensky RP, Reichmann WM, et al. Original research impact of obesity and knee osteoarthritis on morbidity and mortality in older Americans. Ann Intern Med 2011;154:217-66.

3. Zhang Y, Jordan JM. Epidemiology of osteoarthritis. Clin Geriatr Med 2010;26:355-69.

4. Neogi T. The epidemiology and impact of pain in osteoarthritis Osteoarthritis Cartilage 2013;21:1145-53.

5. Finan PH, Buenaver LF, Bounds SC, et al. Discordance between pain and radiographic severity in knee osteoarthritis: findings from quantitative sensory testing of central sensitization. Arthritis Rheum 2013;65:363-72.

6. Neogi T, Frey-Law L, Scholz J, et al. Sensitivity and sensitisation in relation to pain severity in knee osteoarthritis: trait or state? Ann Rheum Dis 2015;74:682-8.

7. Neogi T, Felson D, Niu J, et al. Association between radiographic features of knee osteoarthritis and pain : results from two cohort studies. BMJ 2009;339:b2844.

8. International Association for the Study of Pain Taxonomy Working Group. IASP Taxonomy. Pain terms-2011. 2011. http://www. iasp-pain.org/Content/NavigationMenu/GeneralResourceLinks/\% 250APainDefinitions

9. Woolf CJ. Central sensitization: implications for the diagnosis and treatment of pain. Pain 2011;152:S2-15.

10. Zhang R-X, Ren K, Dubner R. Osteoarthritis pain mechanisms: basic studies in animal models. Osteoarthritis Cartilage 2013;21:1308-15.

11. Arendt-Nielsen $L$, Nie $H$, Laursen MB, et al. Sensitization in patients with painful knee osteoarthritis. Pain 2010;149:573-81.

12. King CD, Sibille KT, Goodin BR, et al. Experimental pain sensitivity differs as a function of clinical pain severity in symptomatic knee osteoarthritis. Osteoarthritis Cartilage 2013;21:1243-52.

13. Gwilym SE, Oag HCL, Tracey I, et al. Evidence that central sensitisation is present in patients with shoulder impingement syndrome and influences the outcome after surgery. J Bone Joint Surg Br 2011;93:498-502.

14. Coombes BK, Bisset L, Vicenzino B. Cold hyperalgesia associated with poorer prognosis in lateral epicondylalgia: a 1-year prognostic study of physical and psychological factors. Clin J Pain 2015;31:30-5.

15. Wylde V, Palmer S, Learmonth ID, et al. The association between pre-operative pain sensitisation and chronic pain after knee replacement an exploratory study. Osteoarthritis Cartilage 2013;21:1253-6.

16. Lundblad $\mathrm{H}$, Kreicbergs $\mathrm{A}$, Jansson $\mathrm{K}$. Prediction of persistent pain after total knee replacement for osteoarthritis. J Bone Joint Surg Br 2008;90:166-71.

17. Wang S-Y, Olson-Kellogg B, Shamliyan TA, et al. Physical therapy interventions for knee pain secondary to osteoarthritis. Ann Intern Med 2012;157:632-44.

18. Weigl M, Angst F, Aeschlimann A, et al. Predictors for response to rehabilitation in patients with hip or knee osteoarthritis: a comparison of logistic regression models with three different definitions of responder. Osteoarthritis Cartilage 2006;14:641-51.

19. Peters TJ, Sanders C, Dieppe P, et al. Factors associated with change in pain and disability over time: a community-based prospective observational study of hip and knee osteoarthritis. $\mathrm{Br} \mathrm{J}$ Gen Pract 2005;55:205-11.

20. Van der Waal JM, Bot SDM, Terwee CB, et al. Course and prognosis of knee complaints in general practice. Arthritis Rheum 2005;53:920-30. 
21. Kittelson AJ, George SZ, Maluf KS, et al. Future directions in painful knee osteoarthritis: harnessing complexity in a heterogeneous population. Phys Ther 2014;94:422-32.

22. Sterling M, Jull G, Vicenzino B, et al. Sensory hypersensitivity occurs soon after whiplash injury and is associated with poor recovery. Pain 2003;104:509-17.

23. Skou ST, Graven-Nielsen T, Lengsoe L, et al. Relating clinical measures of pain with experimentally assessed pain mechanisms in patients with knee osteoarthritis. Scand J Pain 2013;4:111-17.

24. Yarnitsky D, Granot M, Granovsky Y. Pain modulation profile and pain therapy: between pro- and anti-nociception. Pain 2014;155:663-5.

25. Cruz-Almeida $\mathrm{Y}$, Fillingim RB. Can quantitative sensory testing move us closer to mechanism-based pain management? Pain Med 2014;15:61-72.

26. Altman R. Development of criteria for the classification and reporting of osteoarthritis: classification of osteoarthritis of the knee. Arthritis Rheum 1986;29:1039-49.

27. McAlindon TE, Bannuru RR, Sullivan MC, et al. OARSI guidelines for the non-surgical management of knee osteoarthritis. Osteoarthritis Cartilage 2014;22:363-88.

28. Backonja MM, Attal N, Baron R, et al. Value of quantitative sensory testing in neurological and pain disorders: NeuPSIG consensus. Pain 2013;154:1807-19.

29. Rolke R, Baron R, Maier C, et al. Quantitative sensory testing in the German Research Network on Neuropathic Pain (DFNS): standardized protocol and reference values. Pain 2006;123:231-43.

30. Gröne E, Crispin A, Fleckenstein J, et al. Test order of quantitative sensory testing facilitates mechanical hyperalgesia in healthy volunteers. J Pain 2012;13:73-80.

31. Granot M, Weissman-Fogel I, Crispel Y, et al. Determinants of endogenous analgesia magnitude in a diffuse noxious inhibitory control (DNIC) paradigm: do conditioning stimulus painfulness, gender and personality variables matter? Pain 2008:136:142-9.

32. Pham T, van der Heijde D, Altman RD, et al. OMERACT-OARSI initiative: Osteoarthritis Research Society International set of responder criteria for osteoarthritis clinical trials revisited. Osteoarthritis Cartilage 2004;12:389-99.

33. McConnell S, Kolopack P, Davis a M. The Western Ontario and McMaster Universities Osteoarthritis Index (WOMAC): a review of its utility and measurement properties. Arthritis Rheum 2001:45:453-61.

34. Theiler R, Sangha O, Schaeren S, et al. Superior responsiveness of the pain and function sections of the Western Ontario and McMaster Universities Osteoarthritis Index (WOMAC) as compared to the Lequesne-Algofunctional Index in patients with osteoarthritis of the lower extremities. Osteoarthritis Cartilage 1999;7:515-19.

35. Jinks C, Jordan K, Croft P. Measuring the population impact of knee pain and disability with the Western Ontario and McMaster Universities Osteoarthritis Index (WOMAC). Pain 2002;100:55-64.

36. Fischer D, Stewart AL, Bloch DA, et al. Capturing the patient's view of change as a clinical outcome measure. J American Med Assoc 1999;282:1157-62.

37. Ferraz MB, Quaresma MR, Aquino LR, et al. Reliability of pain scales in the assessment of literate and illiterate patients with rheumatoid arthritis. J Rheumatol 1990;17:1022-4.

38. Childs JD, Piva SR, Fritz JM. Responsiveness of the numeric pain rating scale in patients with low back pain. Spine (Phila Pa 1976) 2005;30:1331-4.

39. Thompson LR, Boudreau R, Hannon MJ, et al. The knee pain map: reliability of a method to identify knee pain location and pattern. Arthritis Rheum 2009;61:725-31.

40. Lacey RJ, Lewis M, Jordan K, et al. Interrater reliability of scoring of pain drawings in a self-report health survey. Spine (Phila Pa 1976) 2005;30:E455-8.

41. White DK, Felson DT, Niu J, et al. Reasons for functional decline despite reductions in knee pain: the Multicenter Osteoarthritis Study. Phys Ther 2011;91:1849-56.

42. Jinks C, Jordan KP, Blagojevic M, et al. Predictors of onset and progression of knee pain in adults living in the community. A prospective study. Rheumatology (Oxford) 2008;47:368-74.

43. Hochman JR, Gagliese L, Davis AM, et al. Neuropathic pain symptoms in a community knee OA cohort. Osteoarthritis Cartilage 2011:19:647-54.

44. Hochman JR, Davis AM, Elkayam J, et al. Neuropathic pain symptoms on the modified painDETECT correlate with signs of central sensitization in knee osteoarthritis. Osteoarthr Cartil 2013:21:1236-42.

45. Gonçalves RS, Meireles C, Gil JN, et al. Responsiveness of intermittent and constant osteoarthritis pain (ICOAP) after physical therapy for knee osteoarthritis. Osteoarthritis Cartilage 2012;20:1116-19.
46. Liu A, Kendzerska T, Stanaitis I, et al. The relationship between knee pain characteristics and symptom state acceptability in people with knee osteoarthritis. Osteoarthritis Cartilage 2014;22:178-83.

47. Mayer T, Neblett $\mathrm{R}$, Cohen $\mathrm{H}$, et al. The development and psychometric validation of the Central Sensitization Inventory (CSI). Pain Pract 2012:12:276-85.

48. Neblett R, Cohen H, Choi Y, et al. The Central Sensitization Inventory (CSI): establishing clinically significant values for identifying central sensitivity syndromes in an outpatient chronic pain sample. J Pain 2013;14:438-45.

49. Brooks R. EuroQol: the current state of play. Health Policy 1996;37:53-72.

50. Herdman M, Gudex C, Lloyd A, et al. Development and preliminary testing of the new five-level version of EQ-5D (EQ-5D-5L). Qual life Res 2011;20:1727-36.

51. Fransen M, Edmonds J. Reliability and validity of the EuroQol in patients with osteoarthritis of the knee. Rheumatology (Oxford) 1999:38:807-13.

52. Walk D, Sehgal N, Moeller-Bertram T, et al. Quantitative sensory testing and mapping: a review of nonautomated quantitative methods for examination of the patient with neuropathic pain. Clin $\mathrm{J}$ Pain 2009;25:632-40.

53. Wylde V, Palmer S, Learmonth ID, et al. Somatosensory abnormalities in knee OA. Rheumatology (Oxford) 2012;51 $535-43$.

54. Geber C, Klein T, Azad S, et al. Test-retest and interobserver reliability of quantitative sensory testing according to the protocol of the German Research Network on Neuropathic Pain (DFNS): a multi-centre study. Pain 2011;152:548-56.

55. Wylde V, Palmer S, Learmonth ID, et al. Test-retest reliability of Quantitative Sensory Testing in knee osteoarthritis and healthy participants. Osteoarthritis Cartilage 2011;19:655-8.

56. Anderson RJ, Craggs JG, Bialosky JE, et al. Temporal summation of second pain: variability in responses to a fixed protocol. Eur $J$ Pain 2014;17:67-74.

57. Yarnitsky D, Arendt-Nielsen L, Bouhassira D, et al. Recommendations on terminology and practice of psychophysical DNIC testing. Eur J Pain 2010;14:339.

58. Oono $\mathrm{Y}, \mathrm{Nie} \mathrm{H}$, Matos RL, et al. The inter- and intra-individual variance in descending pain modulation evoked by different conditioning stimuli in healthy men. Scand J Pain 2011;2:162-9.

59. Wolfe $\mathrm{F}, \mathrm{Smythe} \mathrm{H}$, Yunus $\mathrm{M}$, et al. The American College of Rheumatology 1990 criteria for the classification of fibromyalgia: report of the Multicentre Criteria Committee. Arthritis Rheum 1990;33:160-72.

60. Jensen OK, Callesen J, Nielsen MG, et al. Reproducibility of tender point examination in chronic low back pain patients as measured by intrarater and inter-rater reliability and agreement: a validation study. BMJ Open 2013;3:pii:e002532.

61. Van Gool CH, Penninx BW, Kempen Gl, et al. Effects of exercise adherence on physical function among overweight older adults with knee osteoarthritis. Arthritis Rheum 2005:53:24-32.

62. Spetch L, Kolt GS. Adherence to sport injury rehabilitation: implications for sports medicine providers and researchers. Phys Ther Sport 2001;2:80-90.

63. Kolt G, McEvoy J. Adherence to rehabilitation in patients with low back pain. Man Ther 2003;8:110-16.

64. Kolt GS, Brewer BW, Pizzari T, et al. The Sport Injury Rehabilitation Adherence Scale: a reliable scale for use in clinical physiotherapy. Physiotherapy 2007:93:17-22.

65. Cleveland RJ, Luong M-LN, Knight JB, et al. Independent associations of socioeconomic factors with disability and pain in adults with knee osteoarthritis. BMC Musculoskelet Disord 2013;14:297.

66. Weigl M, Angst F, Stucki G, et al. Inpatient rehabilitation for hip or knee osteoarthritis: 2 year follow up study. Ann Rheum Dis 2004;63:360-8

67. Lee CMY, Huxley RR, Wildman RP, et al. Indices of abdominal obesity are better discriminators of cardiovascular risk factors than BMI: a meta-analysis. J Clin Epidemiol 2008;61:646-53.

68. Papassotiropoulos A, Heun R. Detection of subthreshold depression and subthreshold anxeity in the elderly. Int $J$ Geriatr Psychiatry 1999;14:643-51.

69. Johnson SR, Archibald A, Davis AM, et al. Is self-reported improvement in osteoarthritis pain and disability reflected in objective measures? J Rheumatol 2007;34:159-64.

70. Jackson JE. A user's guide to principal components. 2nd edn. John Wiley \& Sons, Inc, 2005.

71. Neziri AY, Curatolo M, Nüesch E, et al. Factor analysis of responses to thermal, electrical, and mechanical painful stimuli supports the 
importance of multi-modal pain assessment. Pain 2011;152:1146-55.

72. Steyerberg EW, Harrell FE, Borsboom GJJ, et al. Internal validation of predictive models. J Clin Epidemiol 2001;54: 774-81.

73. Peduzzi P, Concato J, Kemper E, et al. A simulation study of the number of events per variable in logistic regression analysis. $J$ Clin Epidemiol 1996;49:1373-9.

74. Hay EM, Foster NE, Thomas E, et al. Effectiveness of community physiotherapy and enhanced pharmacy review for knee pain in people aged over 55 presenting to primary care: pragmatic randomised trial. BMJ 2006;333:995.
75. O'Neill S, Manniche C, Graven-Nielsen T, et al. Association between a composite score of pain sensitivity and clinical parameters in low-back pain. Clin J Pain 2014;30:831-8.

76. Creamer P, Lethbridge-Cejku M, Hochberg M. Determinants of pain severity in knee osteoarthritis: effect of demographic and psychosocial variables using 3 pain measures. J Rheumatol 1999;26:1785-92.

77. Mallen CD, Peat G, Thomas E, et al. Prognostic factors for musculoskeletal pain in primary care: a systematic review. $\mathrm{Br} J \mathrm{Gen}$ Pract 2007;57:655-61.

78. Thomas E, Dunn KM, Mallen C, et al. A prognostic approach to defining chronic pain: application to knee pain in older adults. Pain 2008;139:389-97. 